\title{
Determinación de la composición de fases en circona mediante un procedimiento polimórfico simple
}

\author{
A. HERNÁNDEZ-JIMÉNEZ1', F. SÁNCHEZ-BAJO'1, A.L. ORTIZ1', F.L. CUMBRERA² \\ ${ }^{1}$ Departamento de Electrónica e Ingeniería Electromecánica. Escuela de Ingenierías Industriales. ${ }^{2}$ Departamento de Física. Facultad de Ciencias \\ Universidad de Extremadura. 06071 Badajoz. España
}

\begin{abstract}
En este trabajo se propone un método polimórfico para el análisis cuantitativo de fases en la circona. Este procedimiento emplea un sistema de ecuaciones compatible y determinado que relaciona las intensidades de un conjunto de grupos de picos del espectro de difracción con las fracciones en peso de las diferentes fases presentes en la muestra. En dicho sistema, además de las abundancias relativas de las fases, se utilizan como incógnitas un conjunto de parámetros que dan cuenta de la aportación de las colas de los grupos de picos adyacentes al grupo de picos considerado. Este procedimiento se ha aplicado a un difractograma simulado correspondiente a una circona con $50 \%$ de fase cúbica, $25 \%$ de fase tetragonal y $25 \%$ de fase monoclínica y también a una muestra en polvo de circona fabricada mediante técnicas sol-gel.
\end{abstract}

Palabras clave: circona, difracción de rayos $X$, análisis cuantitativo, métodos polimórficos.

\section{Phase composition analysis of zirconia using a simple polimorphic method}

In this work, we propose a polymorphic method for the quantitative analysis in zirconia. This procedure uses a square system of equations relating the intensities of a peaks groups set in the diffraction pattern with the weight fractions of the different phases present in the sample. In this system it is used as unknowns, besides the relative abundance of the phases, a group of parameters that incorporates the contribution from the tails of the groups adjacent to the intensity of a selected group. The described procedure has been applied to a simulated diffractogram corresponding to a composition of zirconia of $50 \%$ of cubic phase, $25 \%$ of tetragonal phase and $25 \%$ of monoclinic phase, and also to a sample of zirconia processed by the sol-gel method.

Keywords: zirconia, X-ray diffraction, quantitative analysis, polymorphic methods.

\section{INTRODUCCIÓN}

La circona $\left(\mathrm{ZrO}_{2}\right)$ en estado puro posee tres variantes polimórficas: monoclínica $(\mathrm{m})$, tetragonal $(\mathrm{t})$ y cúbica $(\mathrm{c})$, estables en diferentes rangos de temperatura (1). La incorporación de elementos tales como Ca, $\mathrm{Mg}, \mathrm{Y}, \mathrm{Ce}$, etc, permite estabilizar a temperatura ambiente las fases de alta temperatura $(\mathrm{t} y \mathrm{c})$, por lo que en la circona es frecuente la coexistencia de dos o más fases, dependiendo del rango del dopado y de los tratamientos mecánicos y / o térmicos. Dada la importancia tecnológica de los materiales cerámicos basados en la circona, y teniendo en cuenta la estrecha relación que existe entre la microestructura del material y sus propiedades, resulta del máximo interés la determinación precisa de las proporciones de las fases presentes. Sin embargo, el análisis cuantitativo mediante difracción de rayos $\mathrm{X}$ de la circona no es una tarea sencilla, debido especialmente al intenso solapamiento entre las reflexiones Bragg de las diferentes fases, así como a la posibilidad de la aparición de fenómenos de orientación preferente.

Se han propuesto diferentes métodos (2-4) para la determinación de las fracciones en peso de las fases presentes en la circona. Estos métodos se basan generalmente en el análisis de las intensidades de un número limitado de reflexiones particulares de las diferentes fases. En la literatura, dichos procedimientos se conocen colectivamente como "métodos polimórficos" y se caracterizan por emplear sólo una parte de la información contenida en el diagrama de difracción completo. Este último es, en cambio, el punto de partida cuando se aplica el método de Rietveld (5) para el análisis cuantitativo en materiales multifásicos. La posibilidad de incluir todas las fases presentes en el material (conocida su estructura cristalográfica) a través de un modelo que se ajusta a los datos experimentales, así como la de tener en cuenta la presencia de fenómenos de textura, extinción, etc, hace del método de Rietveld un pro- cedimiento intrínsecamente superior en el análisis cuantitativo, frente a los métodos polimórficos tradicionales. Sin embargo, la complejidad del método de Rietveld supone un inconveniente cuando se pretende determinar las fracciones en peso de muestras de circona de una forma sencilla y con un tiempo de computación lo más corto posible.

Siguiendo esta idea, en este trabajo se presenta la aplicación en la circona de un método polimórfico basado en el análisis de grupos de picos (en vez de reflexiones individuales) que ha sido propuesto previamente para el análisis cuantitativo en materiales de $\mathrm{SiC}$ (6). Con el objeto de valorar su eficacia, dicho procedimiento se ha aplicado a un diagrama de difracción simulado para una composición de 50\% de fase cúbica, $25 \%$ de fase tetragonal y $25 \%$ de fase monoclínica. Además, el método se ha aplicado al diagrama de difracción de una muestra de circona procesada mediante la técnica sol-gel y la composición obtenida se ha comparado con los resultados del análisis de Rietveld.

\section{DESCRIPCIÓN DEL MÉTODO}

En una muestra que contiene varias fases cristalinas la intensidad observada de la reflexión g de la fase j, $I_{i g^{\prime}}$ es directamente proporcional a la fracción en peso de dicha fase en la muestra (7)

$$
I_{j g}=\frac{K M_{j g} L P_{j g} F_{j g}^{2} \rho}{V_{j}^{2} e^{2 m_{j g}} \mu \rho_{j}} X_{j}=C R_{j g} X_{j}
$$

donde $M_{j g^{\prime}} L P_{j g^{\prime}} F_{j g}$ y $e^{2 m j g}$ son la multiplicidad, el factor de Lorentzpolarización, el factor de estructura y el factor de temperatura de Debye-Waller, respectivamente. Los términos $V_{j}$ y $\rho_{j}$ representan el 
volumen de la celda unidad y la densidad de la fase correspondiente. Finalmente, $\mu$, $\rho$ y $K$ son el coeficiente de absorción lineal, la densidad de la muestra y una constante instrumental, que se incluyen en la constante $\mathrm{C}=\mathrm{K} \rho / \mu$

En el caso de reflexiones que se solapan fuertemente, de forma que no se pueden distinguir individualmente, es preciso considerar grupos de picos cuya intensidad viene definida por:

$$
I_{i}=f+\sum_{j} \sum_{g} I_{j g}
$$

donde $f$ representa la contribución del fondo de intensidades, y los sumatorios se extienden sobre todas las fases y reflexiones individuales que constituyen el grupo $i$.

Considerando, además, la posiblidad de que los grupos de picos solapen entre si, debemos tener en cuenta la contribución a cada grupo debida al solapamiento de grupos vecinos $\beta_{\mathrm{i}} \mathrm{y}$, por tanto:

$$
I_{i}=f+\sum_{j} \sum_{g} I_{j g}+\beta_{i}=f+\sum_{j} \sum_{g} R_{j g} X_{j}^{\prime}+\beta_{i}
$$

donde $X_{j}^{\prime}=C X_{j}$ es un nuevo conjunto de parámetros, a partir de los cuales podemos obtener la composición de la muestra aplicando la condición de normalización $\sum_{j=1}^{n} X_{j}=1$, que implica:

$$
X_{j}=\frac{X_{j}^{\prime}}{\sum_{j=1}^{n} X_{j}^{\prime}}
$$

La introducción de los parámetros $\beta_{i}$ en la ecuación 3 permite usar un sistema con mayor numero de ecuaciones o grupos de picos que de fases y sin embargo compatible y determinado, escogiendo un número de parámetros $\beta_{i}$ igual al número de ecuaciones menos el número de fases menos uno.

La ecuación 3, que es totalmente general, se puede fácilmente particularizar a cualquier material polifásico si se conoce de forma precisa la estructura cristalográfica de las fases presentes en la muestra, que sirve para calcular previamente los coeficientes $R_{j}$.

La selección de los grupos de picos más adecuados para el empleo de este nuevo método polimórfico en muestras de zirconia ha sido una tarea compleja. La primera dificultad fue decidir el número idóneo, dado que se debía conjugar el número máximo de fases presentes con las nuevas incógnitas que aparecen en el método para dar cuenta de los solapamientos entre grupos de picos $\left(\beta_{\mathrm{i}}\right)$. Dos criterios adicionales han sido la elección de los grupos de picos más intensos de la región de bajo ángulo y la inclusión de picos asociados a una única fase.

En este trabajo se han empleado ocho grupos de picos en las posiciones angulares $2 \theta 28.1^{\circ}, 30.2^{\circ}, 31.3^{\circ}, 34.7^{\circ}, 35.1^{\circ}, 50.4^{\circ} .59 .4^{\circ}$ y $59.9^{\circ}$, tres incógnitas correspondientes a las fracciones en peso de las fases (c, $\mathrm{t}, \mathrm{m})$, otra incógnita que da cuenta del fondo, y cuatro coeficientes $\beta_{\mathrm{i}}$ para considerar el posible solapamiento entre grupos de picos vecinos. De esta forma, el sistema resultante es el siguiente:

$\left[\begin{array}{c}I_{2 \theta=28.1^{\circ}} \\ I_{2 \theta=30.2^{\circ}} \\ I_{2 \theta=31.4^{\circ}} \\ I_{2 \theta=34.7^{\circ}} \\ I_{2 \theta=35.1^{\circ}} \\ I_{2 \theta=50.4^{\circ}} \\ I_{2 \theta=59.4^{\circ}} \\ I_{2 \theta=59.9^{\circ}}\end{array}\right]=\left[\begin{array}{cccccccc}0 & 0 & 13.67 & 0 & 0 & 0 & 0 & 1 \\ 28.47 & 29.03 & 0 & 0 & 0 & 0 & 0 & 1 \\ 0 & 0 & 9.74 & 1 & 0 & 0 & 0 & 1 \\ 0 & 2.31 & 0 & 0 & 1 & 0 & 0 & 1 \\ 6.14 & 4.02 & 2.22 & 0 & 0 & 0 & 0 & 1 \\ 15.99 & 5.71 & 1.91 & 0 & 0 & 1 & 0 & 1 \\ 0 & 4.03 & 0 & 0 & 0 & 0 & 0 & 1 \\ 11.02 & 7.14 & 1.14 & 0 & 0 & 0 & 1 & 1\end{array}\right]\left[\begin{array}{c}X_{C}^{\prime} \\ X_{T}^{\prime} \\ X_{M}^{\prime} \\ \beta_{2 \theta=31.4^{\circ}} \\ \beta_{2 \theta=34.7^{\circ}} \\ \beta_{2 \theta=50.4^{\circ}} \\ \beta_{2 \theta=59.9^{\circ}} \\ f\end{array}\right]$

En la figura 1 se muestra un espectro de difracción simulado, indicando la posición de los grupos de picos empleados en este estudio junto con las fases que contribuyen a cada uno de ellos.

\section{PROCEDIMIENTO EXPERIMENTAL}

\subsection{Obtención del difractograma simulado.}

Los detalles del procedimiento de simulación del perfil de difracción de rayos $X$ se han presentado en un trabajo previo (8). Brevemente, los perfiles individuales de las fases cúbica, tetragonal y monoclínica se generaron a partir de sus estructuras cristalográficas considerando radiación $\mathrm{CuK} \alpha$ y un difractómetro con geometría Debye-Scherrer. El perfil completo se obtuvo como suma ponderada de dichos perfiles individuales asumiendo una composición en peso con un $50 \%$ de fase cúbica, $25 \%$ de tetragonal y $25 \%$ de monoclínica. Con el fin de reproducir de forma realista un perfil experimental de difracción de rayos $X$, se añadieron una contribución instrumental, un fondo de intensidades modelado mediante una función polinómica, y un ruido estadístico asumiendo una distribución de Poisson. El rango de perfil simulado se extendió desde $25^{\circ}$ hasta $65^{\circ}$ con un paso de $0.01^{\circ}$. Finalmente, el espectro de difracción fue analizado utilizando el método polimórfico ya descrito.

\subsection{Caracterización mediante difracción de rayos $X$ de la muestra real de circona}

Se empleó una muestra de circona dopada con un $6 \mathrm{~mol} \%$ de $\mathrm{CaO}$, obtenida en forma de polvo por un proceso sol-gel (9) y calcinada posteriormente a $700{ }^{\circ} \mathrm{C}$ durante 5 horas. Los datos de difracción de rayos $\mathrm{X}$ se obtuvieron en un difractómetro Philips PW-1800, empleando radiación $\mathrm{CuK} \alpha$ con un monocromador secundario de grafito. Las condiciones de trabajo fueron $40 \mathrm{kV}$ y $35 \mathrm{~mA}$. El difractograma se recogió sobre un intervalo angular $(2 \theta)$ desde $25^{\circ}$ hasta $90^{\circ}$ con un paso de $0.05^{\circ}$ y un tiempo de conteo de 5 segundos.

La determinación de las proporciones de las diferentes fases presentes en la muestra se ha realizado usando el método polimórfico descrito anteriormente y el método de Rietveld mediante el programa FullProf (10) .

\section{RESULTADOS Y DISCUSIÓN}

La composición de fases obtenida tanto para la muestra simulada como para la muestra obtenida vía sol-gel se muestra en la figura 2. En la figura 2a están representados los resultados correspondientes a la muestra simulada comparando la composición obtenida mediante el método polimórfico con la composición simulada. Puede observarse que las fracciones en peso obtenidas concuerdan razonablemente bien con los valores utilizados en la generación del perfil. Las mayores discrepancias se dan para las fases cúbica y tetragonal $(6.4 \%$ y 5.6

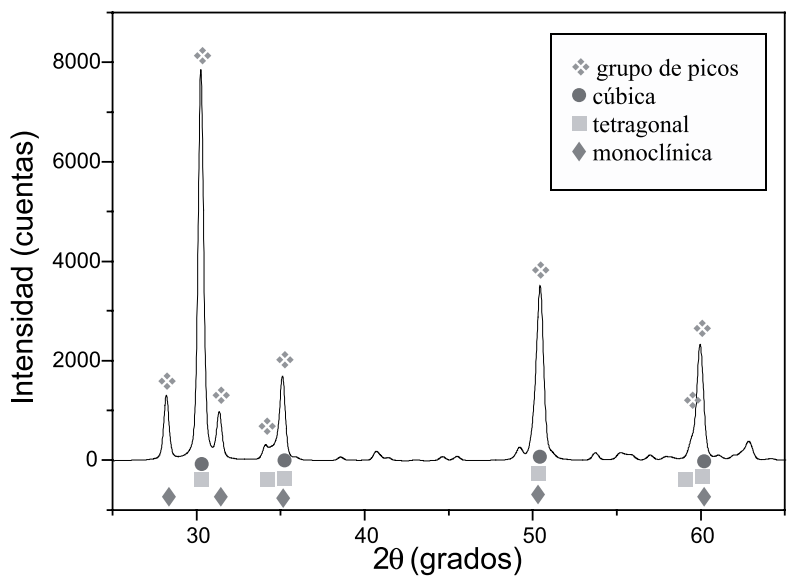

Figura 1. Grupos de picos empleados e identificación de las fases de la circona. 
$\%$ en peso, respectivamente). Este resultado es lógico teniendo en cuenta que en los grupos de picos estudiados existe un muy fuerte solapamiento entre las fases tetragonal y cúbica. Además el grupo de picos más intenso $\left(2 \theta=30.2^{\circ}\right)$ presenta únicamente contribuciones de las fases t y c con coeficientes $R_{j g}$ muy similares, lo que ocasiona una cierta inestabilidad del sistema de ecuaciones.

En figura $2 \mathrm{~b}$ se presentan los resultados correspondientes a la muestra de circona dopada con calcia. Dicha muestra no presenta fase cúbica apreciable según el método de Rietveld, mientras que el método polimórfico determina una pequeña fracción de dicha fase, que viene acompañada de una reducción similar en el contenido de fase tetragonal. La explicación del fenómeno podría ser similar a la expuesta para el caso de la muestra simulada.

Finalmente, en la figura 3 se presenta el resultado del ajuste de Rietveld para la muestra de circona dopada con calcia. Como puede apreciarse, existe una buen acuerdo entre el perfil experimental y el calculado y, por tanto, resulta razonable la hipótesis de que los resultados del ajuste pueden considerarse cercanos a los valores reales de la composición de la muestra, permitiendo valorar de forma aproximada la eficacia del método polimórfico en dicha muestra.

La fracción en peso de la fase monoclínica es casi igual a la original en el caso de la muestra simulada. Por otra parte, los resultados obtenidos para la muestra de $\mathrm{Ca}-\mathrm{ZrO}_{2}$ mediante el método de Rietveld y el método polimórfico son bastante similares. Este resultado se debe a que dos grupos de picos muy poco solapados están constituidos exclusivamente por fase monoclínica, lo que permite discriminar de forma más precisa el contenido de dicha fase.

\section{CONCLUSIONES}

En este estudio se ha propuesto un nuevo método polimórfico para el análisis cuantitativo en la circona parcialmente estabilizada, basado en el análisis de grupos de picos del diagrama de difracción de rayos X. El procedimiento se ha aplicado a un difractograma simulado correspondiente a una composición de $50 \%$ de fase cúbica, $25 \%$ de fase tetragonal y $25 \%$ de fase monoclínica, y a una muestra de circona dopada con calcia. Los resultados muestran un acuerdo razonable entre las fracciones en peso obtenidas con el método y las originales para la muestra simulada. Para la Ca- $\mathrm{ZrO}_{2^{\prime}}$ la comparación con los datos procedentes de la aplicación del método de Rietveld muestra discrepancias algo más acusadas en las composiciones de fase cúbica y tetragonal, debidas probablemente al intenso solapamiento entre las reflexiones de ambas fases en la región de bajo ángulo del espectro de difracción.

\section{AGRADECIMIENTOS}

Los autores de este trabajo desean agradecer la ayuda recibida a través del proyecto MAT2001-0799 (Plan Nacional de Investigación Científica, Desarrollo e Innovación Tecnológica 2000-2003).

\section{BIBLIOGRAFÍA}

1. E.C. Subbarao. "Zirconia-an overview", pp. 1-24 en Advances in Ceramics vol. 3: Science and Technology of Zirconia. First International Conference on the Science and Technology of Zirconia, Cleveland, Ohio (EE.UU.), Junio 1980. Ed. A.H. Heuer y L.W. Hobbs, The American Ceramic Society, Inc., Columbus, Ohio 1981.

2. D.L. Porter, A.H. Heuer. "Microstructural Development in MgO-Partially Stabilized Zirconia (Mg-PSZ)". J. Am. Ceram. Soc. 62 [5-6] 298-305 (1979).

3. R.A. Miller, J.L. Smialek, R.G. Garlick. "Phase stability in plasma-sprayed, partially stabilized zirconia-yttria", pp. 241-253 en Advances in Ceramics vol. 3: Science and Technology of Zirconia. First International Conference on the Science and Technology of Zirconia, Cleveland, Ohio (EE.UU.), Junio

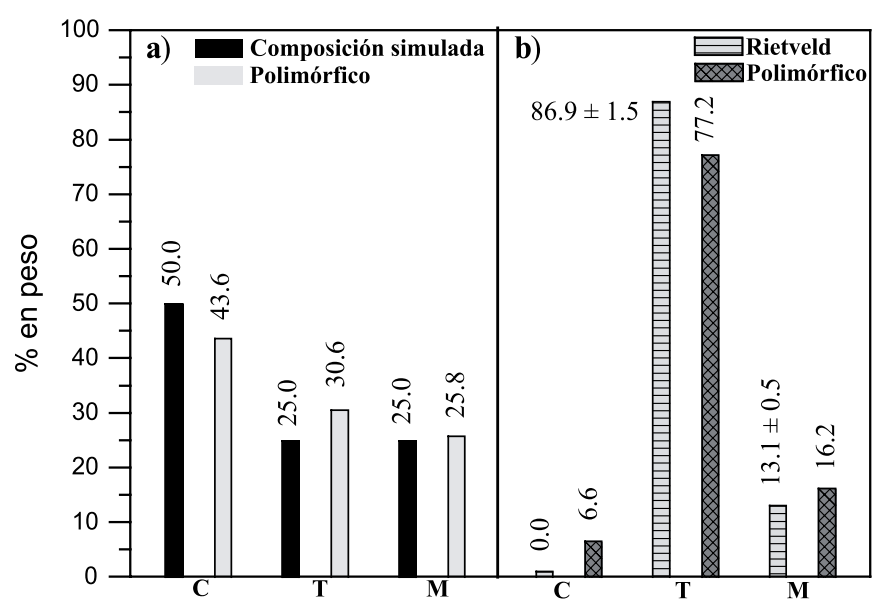

Figura 2. Composición de las muestras simulada (a) y real (b).

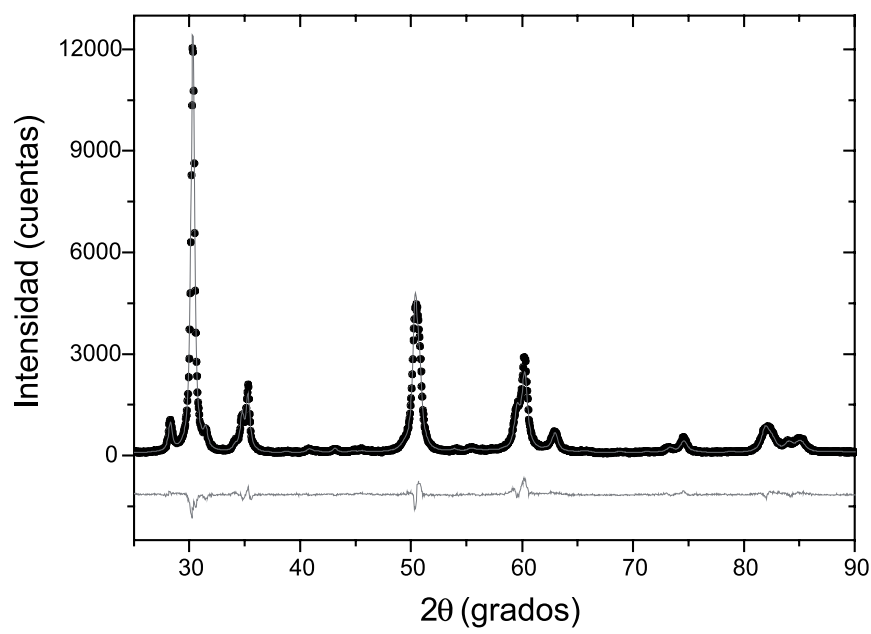

Figura 3. Difractograma de la muestra de circona dopada con calcia. Los puntos representan los datos de difracción de rayos $\mathrm{X}$ y la línea sólida el espectro calculado mediante el modelo de mejor ajuste obtenido tras la aplicación del método de Rietveld. La línea sólida inferior constituye el perfil de diferencias (intensidad observada menos calculada).

1980. Ed. A.H. Heuer y L.W. Hobbs, The American Ceramic Society, Inc., Columbus, Ohio 1981.

4. C.J. Howard, E.H. Kisi. "'Polymorph Method" Determination of Monoclinic Zirconia in Partially Stabilized Zirconia Ceramics". J. Am. Ceram. Soc. 73 [10] 3096-3099 (1990).

5. H.M. Rietveld. "A Profile Refinement Method for Nuclear and Magnetic Structures". J. Appl. Cryst. 2, 65-71 (1969).

6. A. Hernández-Jiménez, A.L. Ortiz, F. Sánchez-Bajo, F.L. Cumbrera. “A simple, accurate and effective polymorphic method to determine phase compositions of SiC-based ceramics", aceptado para su publicación en J. Eur. Ceram. Soc..

7. H.P. Klug, L.E. Alexander. “X-ray diffraction procedures for polycrystalline and amorphous materials". John Wiley and Sons, New York (1974).

8. A.L. Ortiz, F. Sánchez-Bajo, N.P. Padture, F.L. Cumbrera, F. Guiberteau. "Quantitative Polytype-Composition Analyses of $\mathrm{SiC}$ Using X-ray Diffraction: A Critical Comparison between the Polymorphic and the Rietveld Methods". J. Eur. Ceram. Soc. 21 [9] 1237-1248 (2001).

9. M.C. Caracoche, P.C. Rivas, M.M. Cervera, R. Caruso, E. Benavídez, O. de Sanctis, M.E. Escobar. " Zirconium Oxide Structure Prepared by the Sol-Gel Route: I, The Role of the Alcoholic Solvent.". J. Am. Ceram. Soc. 83 [2] 377384 (2000).

10. J. Rodríguez-Carvajal. "FULLPROF: A Program for Rietveld Refinement and Pattern Matching Analysis". pp. 127 en Abstract of the Satellite Meeting on Powder Diffraction of the XV Congress of the IUCr. Toulouse, Francia (1990).

Recibido: 01.02 .03

Aceptado: 30.11 .03 